\title{
EGFR and ErbB2 Differentially Regulate Raf-1 Translocation and Activation
}

\author{
Lianfeng Zhang, Mary Bewick, and Robert M. Lafrenie \\ Division of Tumor Biology, Northeastern Ontario Regional Cancer Centre, Sudbury, Ontario, Canada
}

\begin{abstract}
SUMMARY: Epidermal growth factor receptor (EGFR) and HER-2/ErbB2 are members of the Erb family of signaling receptors. ErbB2 is overexpressed in many different cancers and has been linked to enhanced malignancy of tumors. We have examined the cellular translocation of Raf-1 during EGF-dependent signal transduction in two breast tumor cell lines, BT20 and SKBR3. Treatment of BT-20 breast cancer cells, which express EGFR, with EGF resulted in rapid (5 minutes) accumulation of EGFR and Raf-1 into plasma membrane-associated endocytotic vesicles. However, at later time points (30 minutes) only EGFR was endocytosed and Raf-1 dissociated from the plasma membrane and was found in the cytosol. In SKBR3 breast cancer cells, which express high levels of EGFR and ErbB2, treatment with EGF also resulted in rapid accumulation of EGFR and Raf-1 into endocytotic vesicles, but EGFR endocytosis was inhibited and Raf-1 remained associated with the plasma membrane for a prolonged period. The role of ErbB2 in the retention of Raf-1 at the plasma membrane was confirmed in BT-20 cells transfected with ErbB2. BT-20 cells expressing ErbB2 and treated with EGF retained Raf-1 at the plasma membrane for prolonged periods, whereas Raf- 1 rapidly dissociated from the plasma membrane in EGF-stimulated cells transfected with a control vector. The presence of Raf- 1 at the plasma membrane correlated with activation of Raf-1 and MAP kinase. Cells that expressed ErbB2 and treated with EGF showed prolonged activation of Raf-1 and MAP kinase compared with cells that expressed low levels of ErbB2. These results suggest that expression of ErbB2 promoted retention of Raf-1 in the plasma membrane, resulting in prolonged activation of the MAP kinase cascade, which may contribute to enhanced malignancy in ErbB2-expressing cancers. (Lab Invest 2002, 82:71-78).
\end{abstract}

$E$ pidermal growth factor receptor (EGFR) and HER2/ErbB2 (also known as neu) are members of the Erb family of receptor tyrosine kinases (RTK). All members of this family, which include EGFR, HER2/ ErbB2, HER3/ErbB3, and HER4/ErbB4, are singlechain membrane proteins that have significant sequence homology to one another (Earp et al, 1995). The RTK are formed by homodimerization or heterodimerization of the Erb family monomers (Heldin, 1995). ErbB2 is an important signaling partner for the EGFR and it is expressed in several human cancers, including breast, ovarian, lung, gastric, and prostate (Afify et al, 1999; Nakajima et al, 1999; Polkowski et al, 1999; Ross and Fletcher, 1999; Schwartz et al, 1999; Slamon et al, 1989). Amplification of the ErbB2 gene and/or overexpression of the ErbB2 protein has been found in $10 \%$ to $40 \%$ of human breast cancers. Patients whose tumor cells overexpress ErbB2 have a higher rate of relapse and shorter survival time compared with patients without ErbB2 overexpression. Overexpression of ErbB2 has also been correlated with lymph node metastasis in patients with breast cancer (Bitran et al, 1996; Lonn et al, 1995; Ross et al, 1999; Slamon et al, 1987, 1989). Evidence from basic and clinical research studies suggest that ErbB2 is

Received October 3, 2001

Address reprint requests to: Dr. Robert Lafrenie, Northeastern Ontario Regional Cancer Centre, 41 Ramsey Lake Road, Sudbury, ON, Canada, P3E 5J1.E-mail:rlafrenie@neorcc.on.ca involved in initial transformation and in progression to metastasis (Lacroix et al, 1989; Tan et al, 1997).

The Raf- 1 serine/threonine kinase is a key regulator in many growth and developmental pathways. The regulation of Raf- 1 is an intricate multistep process involving changes in Raf-1 subcellular localization and changes in protein-protein interactions in response to phosphorylation events (Marshall, 1994; Moelling et al, 1984; Morrison and Cutler, 1997). Deletion analysis confirms that truncation of Raf-1, by removal of its $\mathrm{N}$-terminus, generates a constitutive active protein that can transform normal cells (Heidecker et al, 1990; Stanton et al, 1989). In fact, some mutants of Raf-1 have been shown to be viral oncogenes (lkawa et al, 1988; Shimizu et al, 1985).

Activated EGFR and ErbB2 can initiate the Ras cascade resulting in activation of MAP kinase (Cantley et al, 1991; Crews and Erikson, 1993; Graus-Porta et al, 1995; Janes et al, 1994). Translocation of Raf-1 to the plasma membrane after EGF/EGFR binding is associated with the interaction of Raf-1 with Ras that controls Raf-1 phosphorylation (Satoh et al, 1992; Vojtek et al, 1993). Once associated with the membrane, Raf- 1 is activated and becomes available to phosphorylate MEK, the next kinase in the MAP kinase cascade. Raf-1 is thought to be involved in the complex that forms around activated EGFR in caveolae on the cell surface (Mineo et al, 1996). Binding of EGF to EGFR rapidly induces clustering and internalization of the ligand-receptor complexes, ultimately resulting in lysosomal degradation of both EGF and its receptor (Carpenter, 1987). Therefore, the endocytotic pathway 
provides a mechanism for the gradual attenuation of plasma membrane signaling complexes and could result in the down-regulation of Raf-1 signaling. However, it is not clear if Raf-1 is internalized with EGFR in endosomes after EGF binding. It is also not known if there is any difference in Raf-1 localization after interaction of EGF with EGFR or ErbB2. In this study we have used green fluorescence protein (GFP)-flagged Raf-1 (Raf-GFP) and cell fractionation to examine the translocation of Raf-1 and differential interactions with EGFR or ErbB2 in response EGF treatment.

\section{Results \\ Translocation of Raf-1 to the Membrane and Endosomes in Response to EGF}

BT20 breast cancer cells transiently transfected with pRaf-GFP were treated with EGF for 5 to 60 minutes to determine if Raf-1 was internalized with EGFR in endosomes (Fig. 1, A to F). Binding of EGF to the EGFR rapidly induced EGFR clustering on the plasma. RafGFP was also recruited into clusters on the plasma membrane, which appeared to colocalize with aggre-

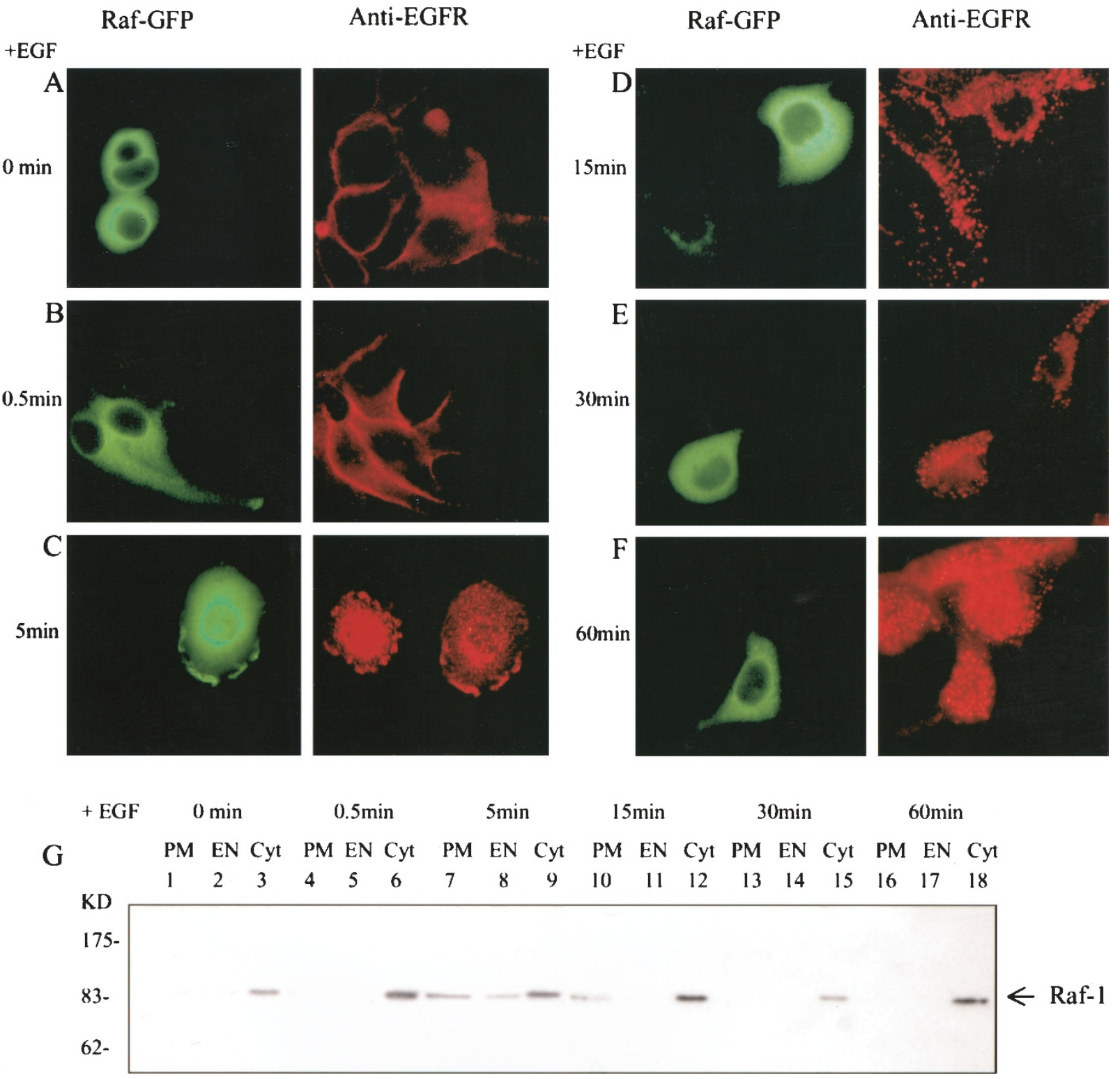

Figure 1.

Localization of Raf-1 and epidermal growth factor receptor (EGFR) by double fluorescence and subcellular fraction analysis. BT20 cells transfected with green fluorescence protein (GFP)-flagged Raf-1 (Raf-GFP) and treated with EGF for various durations were fixed by incubation in methanol. The cells were stained with goat polyclonal anti-EGFR antibody and detected by using an anti-goat IgG-rhodamine secondary antibody (left panels). Raf-1-GFP localization was detected by measuring the distribution of GFP fluorescence (right panels). Cells were treated with EGF for 0 minutes (A), 0.5 minutes (B), 5 minutes (C), 15 minutes (D), 30 minutes (E), and 60 minutes (F). BT-20 cells treated with EGF were subjected to subcellular fractionation and the plasma membrane (PM), endosomal (EN), and cytosolic (Cyt) fractions were normalized for protein and subjected to immunoblot analysis using the mouse monoclonal anti-Raf-1 antibody (G). Lanes 1, 4, 7, 10, 13, and 16 were fractions of PM, lanes 2, 5, 8,11, 14, and 17 were fractions of EN, and lanes 3, 6, 9, 12, 15, and 18 were fractions of Cyt. 
gated EGFR after 5 minutes of EGF stimulation (Fig. 1C, left versus right panel). However, Raf-GFP dissociated from these clusters on the plasma membrane within 15 minutes of EGF stimulation (Fig. 1, D and E, left panels), whereas EGFR was internalized to endosomes and then translocated to the perinuclear area (Fig.1, D to F, right panels). Raf-1-GFP did not internalize with EGFR in the endosomes. Subcellular fractionation analysis of untransfected BT-20 cells also showed that native Raf- 1 was localized in the plasma membrane fraction 5 to 15 minutes after EGF stimulation, but was not found in the plasma membrane fraction 30 or 60 minutes after EGF treatment. Raf-1 was also found in the endosome fraction 5 minutes after EGF stimulation (Fig. 1G). This suggested that Raf-1 was associated with early endosome formation.

\section{Raf-GFP is Retained on the Plasma Membrane Longer in SKBR3 than BT-20 Cells}

BT-20 and SKBR3 breast cancer cells express different ratios of the EGFR and ErbB2 monomers (Fig. 2, G and H). EGFR was highly expressed in both BT-20 and SKBR3 cells. ErbB2 was highly expressed in SKBR3 cells, but was expressed at low levels in BT-20 cells (Fig. $2 \mathrm{H})$. The translocation of Raf-GFP in BT-20 and SKBR3 cells after EGF treatment was compared by determining the localization of GFP-dependent fluorescence (Fig. 2, A to F). Raf-GFP was recruited to clusters on the plasma membrane of both cell types that had a similar localization to aggregated EGFR 5 minutes after EGF treatment. In BT-20 cells, Raf-GFP was translocated to the cytosol 15 minutes after EGF treatment (Fig. 2D, left panel). In contrast, in SKBR3 cells, Raf-GFP remained localized on the plasma membrane for more than 60 minutes (Fig. 2, $\mathrm{C}$ to $\mathrm{F}$, right panels).

Subcellular fractionation of BT-20 and SKBR3 cells after EGF treatment was also used to analyze the localization of Raf- 1 in the plasma membrane fraction (Fig. 2, I and J). A small amount of Raf-1 was present in the plasma membrane of untreated BT-20 and SKBR3 cells (EGF $=0$ minutes). The amount of Raf- 1 present in the plasma membrane fraction of both BT-20 and SKBR3 cells increased dramatically after treatment with EGF for 0.5 minutes and 5 minutes. A larger amount of Raf-1 was found in the plasma membrane fraction of SKBR3 cells than in BT-20 cells immediately after EGF treatment. In BT-20 cells, the amount of Raf-1 in the plasma membrane fraction declined to control levels within 15 minutes after EGF treatment and was undetectable at 30 and 60 minutes after treatment. In SKBR3 cells, which express high levels of ErbB2, Raf-1 was retained in the plasma membrane fraction for 60 minutes.

\section{Expression of Erb2 Delayed Dissociation of Raf-1 from the Plasma Membrane in Response to EGF}

BT-20 cells expressed high levels of EGFR but expressed very low levels of ErbB2. To determine if ErbB2 was mechanistically involved in the retention of Raf- 1 in the plasma membrane after EGF treatment,
BT-20 cancer cells were transiently transfected with pcDNAErbB2 and Raf-1 localization examined. BT-20 cells transfected with pcDNAErbB2 expressed approximately three times as much ErbB2 protein as BT-20 cells transfected with the pcDNA control vector in immunoblot analysis using an anti-ErbB2 antibody (Fig. 3A). Cells transfected with pcDNA or pcDNAErbB2 were treated with EGF for different durations and the amount of Raf-1 present in the plasma membrane fractions was determined. BT-20 cells transfected with pcDNAErbB2 and treated with EGF retained Raf-1 in the plasma membrane fraction for 60 minutes, whereas cells transfected with pcDNA downregulated the level of Raf-1 in the plasma membrane by 30 minutes after EGF treatment (Fig. 3, B to D).

\section{Expression of Erb2 Increased the Length of Signal from Raf-1 to MAP Kinase}

EGF-dependent translocation of Raf-1 to the plasma membrane is required for interaction with Ras and for Raf-1 phosphorylation and activation of the MAP kinase pathway. Because increased levels of ErbB2 were associated with retention of Raf- 1 in the plasma membrane after EGF treatment, the effects of ErbB2 on the kinetics of EGF-dependent Raf-1 and MAP kinase activation were determined. Activated Raf-1 and MAP kinase were detected in the total cell lysates by immunoblot analysis with an anti-phospho-Raf-1 antibody (Fig. 4A) and an anti-phospho-p42/44-MAP kinase antibody (Fig. 4B). In BT-20 cells transiently transfected with the pcDNA control vector and treated with EGF, Raf-1 phosphorylation was maximal at 15 minutes and was undetectable at 60 minutes after EGF treatment, and MAP kinase phosphorylation was maximal at 15 minutes and undetectable at $90 \mathrm{~min}-$ utes. In contrast, BT-20 cells transiently transfected with pcDNAErbB2 showed prolonged activation of Raf-1 and MAP kinase. Phosphorylated Raf-1 and phosphorylated MAP kinase were present for at least 90 minutes after EGF treatment.

\section{Discussion}

The translocation of Raf-1 between the plasma membrane and cytosol after EGF stimulation of cells appears to be mechanistically important in endocytotic down-regulation of MAP kinase signal transduction. Raf-1 is recruited to plasma membrane caveolae and is involved in a complex that includes activated EGFR (Mineo et al, 1996). Raf-1 becomes activated after EGF treatment and then becomes available to phosphorylate MEK, the next kinase in the MAP kinase cascade (Satoh et al, 1992; Vojtek et al, 1993). Determining the kinetics of Raf-1 dissociation from the complexes formed by the EGFR homodimer or EGFR/ErbB2 heterodimer after EGF treatment should help define the downstream regulation of EGF-transduction signals.

In the current experiments we have used Raf-GFP to show that Raf- 1 was recruited to the plasma membrane in proximity to aggregated EGFR 5 minutes 


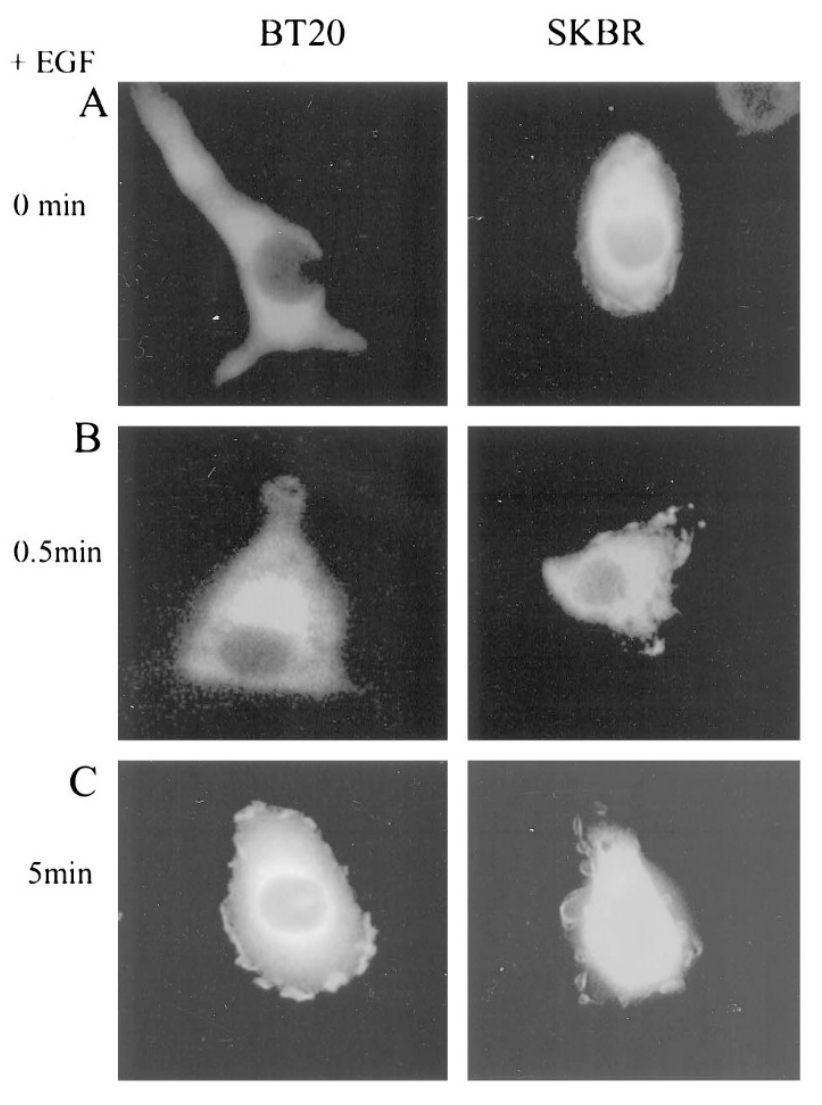

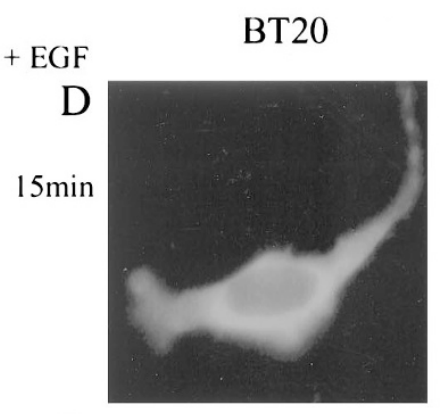

\section{SKBR}
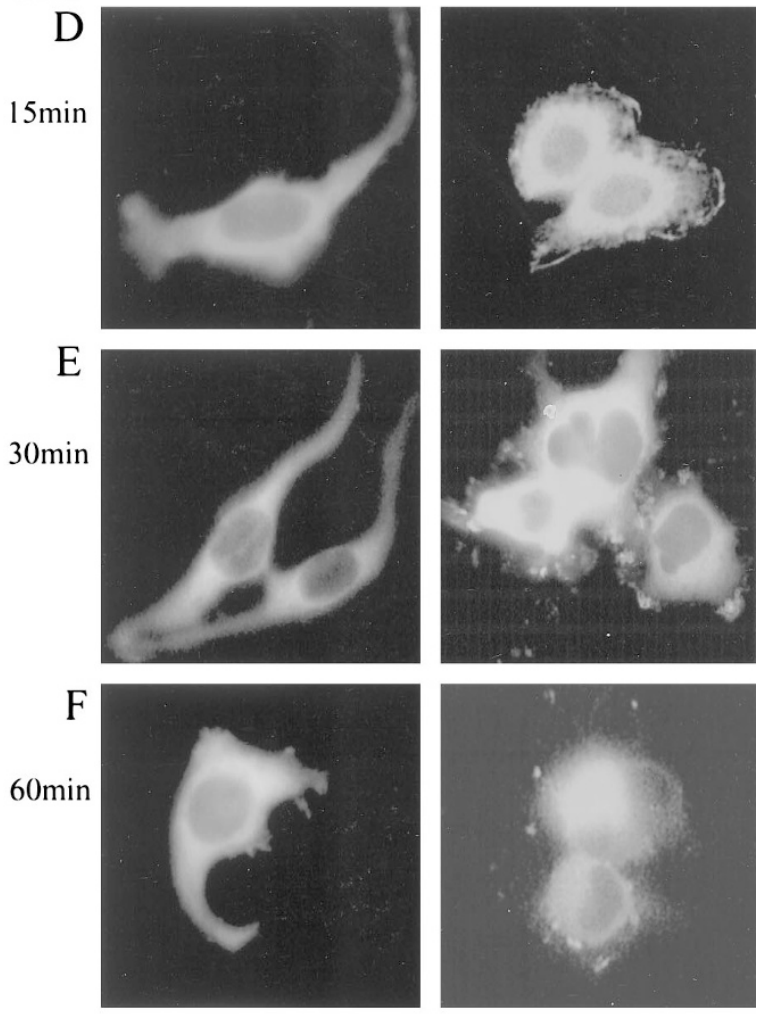

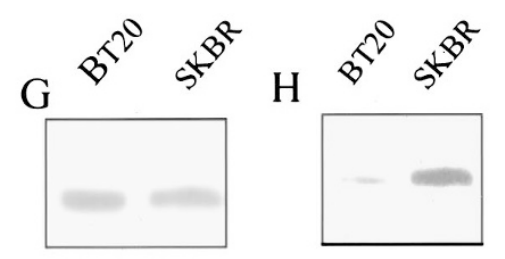

Blot:anti-EGFR Reblot:anti-ErbB2

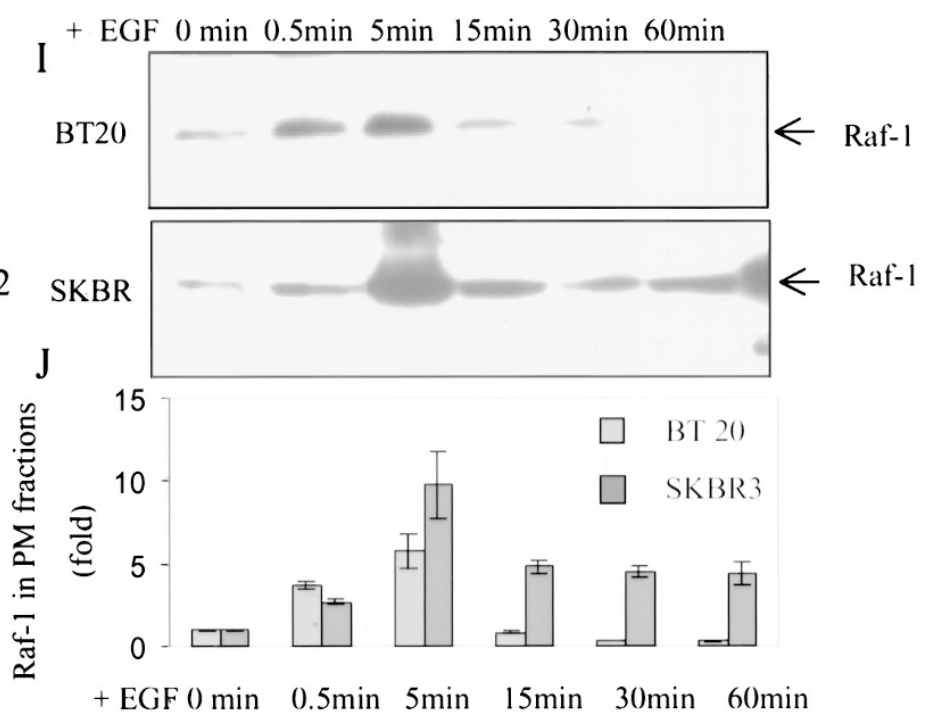

Figure 2.

Localization of Raf-1 in BT20 and SKBR3 cells using Raf-GFP fluorescence and subcellular fractionation analysis in response EGF. BT20 and SKBR3 cells were transiently transfected with pRaf-GFP, treated with EGF for 0 minutes (A), 0.5 minutes (B), 5 minutes (C), 15 minutes (D), 30 minutes $(E)$, or 60 minutes (F) and fixed with methanol. Raf-1 localization was detected by the fluorescence of Raf-GFP. Total lysates of BT20 and SKBR3 were subjected to immunoblot analysis with polyclonal goat anti-EGFR (G) and after stripping, the blot was reprobed with polyclonal goat anti-ErbB2 (H). BT20 and SKBR3 cells were treated with EGF for 0 minutes, 0.5 minutes, 5 minutes, 15 minutes, 30 minutes, or 60 minutes and subjected to subcellular fractionation. Samples of PM fraction were subjected to immunoblot analysis with monoclonal mouse anti-Raf-1 antibody to detect the amount of Raf-1 in the PM fraction (I). The relative intensity of Raf-1 anchored on PM fractions in BT20 and SKBR3 cells responding EGF stimulation was assayed by densitometric analysis of autoradiographs. The density of Raf- 1 staining at 0 minutes was set as $1(\mathrm{~J})$.

after treatment with EGF. Subcellular fractionation analysis further revealed that endogenous Raf- 1 localized to the plasma membrane fraction 5 to 15 minutes after EGF stimulation and then dissociated from the plasma membrane after 15 minutes. Raf- 1 was also found in the early-stage endosomal fraction 5 minutes after stimulation. This suggests that Raf- 1 is associated with early endosome formation and dissociates from endosomes soon after the stimulation with EGF and before EGFR endocytosis. 


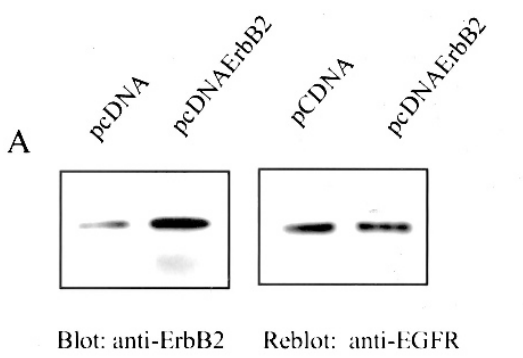

$+\mathrm{EGF} 0 \mathrm{~min} 0.5 \mathrm{~min} 5 \mathrm{~min} 15 \mathrm{~min} 30 \mathrm{~min} 60 \mathrm{~min}$

B

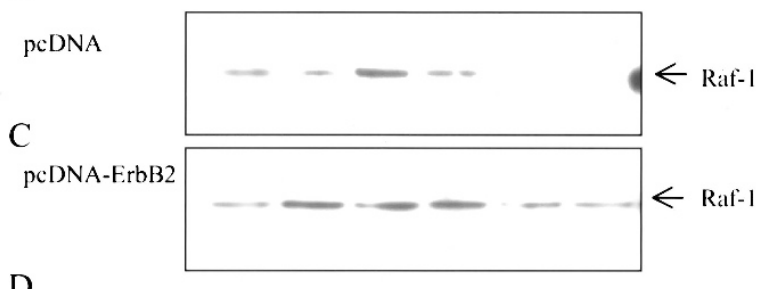

D

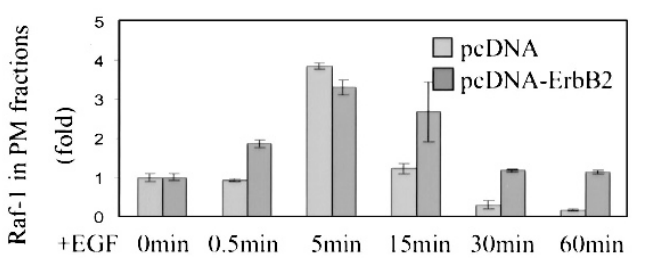

Figure 3.

Transient expression of ErbB2 in BT20 cells increases retention of Raf- 1 in the plasma membrane. BT20 cells were transiently transfected with pcDNA control vector or pcDNAErbB2. Total lysates of the transfected BT20 cells were subjected to immunoblot analysis with polyclonal goat anti-ErbB2 (A, left), and then reprobed using polyclonal goat anti-EGFR (A, right). BT20 cells transfected with pcDNA (B) or pcDNAErbB2 (C) were treated with EFG and subjected to subcellular fractionation. The PM fractions were separated and subjected to immunoblot analysis with the monoclonal mouse anti-Raf-1 antibody. The relative intensity of Raf-1 anchored on PM fractions responding to EGF stimulation was assayed by densitometric analysis of autoradiographs. The density of Raf-1 staining at 0 minutes was set as 1 (D).

EGF stimulates the formation of heterodimers between monomeric EGFR and monomeric ErbB2 (Goldman et al, 1990; Wada et al, 1990) and subsequent tyrosine phosphorylation of ErbB2 (Holmes et al, 1992; Karunagaran et al, 1996). Both the EGFR and ErbB2 monomers are expressed in SKBR3 cells and therefore the EGFR/ErbB2 heterodimer was highly expressed in SKBR3 cells. In contrast, BT-20 cells expressed EGFR but very low levels of ErbB2 and therefore the EGFR homodimer was predominant in BT-20 cells as was previously shown (Wang et al, 1999). To compare Raf-1 translocation in response to EGF stimulation, SKBR3 and BT-20 cells were transfected with Raf-GFP to trace the translocation of Raf-1. The results of these studies showed that the presence of EGFR/ErbB2 in the plasma membrane was associated with retention of Raf- 1 at the plasma membrane and suggest that the impaired endocytosis of EGFR/ErbB2 was associated with impaired dissociation of Raf- 1 from the plasma membrane. BT-20 cells transiently transfected with the ErbB2 gene also showed retention of Raf- 1 in the plasma membrane after EGF treatment. This result supports the idea that ErbB2 delays the dissociation of Raf- 1 from the plasma membrane. Translocation of Raf- 1 to the cell membrane is required for Raf-1 activation (Dent et al, 1996; Leevers et al, 1994). Activated Raf-1 can phosphorylate MEK, and MEK can in turn activate MAP kinase, which results in stimulation of cell growth (Lange-Carter et al, 1993). The results of the current experiments showed that the presence of Raf-1 in the plasma membrane fraction correlated with the activation of Raf- 1 . Thus, those cells that expressed higher levels of ErbB2 and retained Raf-1 in the plasma membrane did not down-regulate Raf-1 activity, resulting in prolonged activation of Raf-1 after EGF stimulation. Further, the continued activation of Raf-1 was associated with an increase in the duration of MAP kinase activation after EGF treatment. Therefore, it appears that retention of Raf-1 in the plasma membrane promotes sustained activation of EGF-dependent activation of mitogenic signals and this difference is dependent on the signaling receptor for EGF.

EGFR is rapidly endocytosed in response to EGF stimulation (Carpenter, 1987). Rapid internalization and lysosomal degradation results in immediate and sustained down-regulation of receptor activity (Sorkin and Waters, 1993). Thus, receptor endocytosis provides a mechanism to shut off EGF-dependent signaling. However, ErbB2 overexpression decreases the rate of receptor endocytosis and EGF dissociation, resulting in a delay in receptor inactivation (Karunagaran et al, 1996; Lenferink et al, 1998). Previous work also showed that EGFR endocytosis was inhibited in $87 \%$ of BT-20 cells microinjected with an ErbB2 expression plasmid, whereas EGFR endocytosis was inhibited in only $8 \%$ of the BT20 cells microinjected with LacZ control plasmid. This work suggested clearly that the endocytosis of EGFR is inhibited by the expression of ErbB2 in BT-20 cells (Wang et al, 1999). Thus, ErbB2 expression may cause an increase in the duration of signal transduction in response to EGF treatment. This idea is in agreement with our current results that showed ErbB2 had a tendency to maintain Raf-1 at the plasma membrane where it was anchored and activated. Breast cancer cell lines such as SKBR3, MDA-MB-453, and BT474, which express high levels of ErbB2 and the EGFR/ErbB2 heterodimer, show impairment in EGF-induced EGFR endocytosis (Wang et al, 1999). In SKBR3 cells, ErbB2 is not degraded in response to EGF (King et al, 1988) and the continued presence of ErbB2 may provide a mechanism to increase the duration of Raf- 1 signaling and promote malignant behavior.

\section{Materials and Methods}

\section{Construction of Raf-GFP and ErbB2}

The full-length Raf-1 (residues 1-648) fragment was generated by PCR amplification using pRaf-1 (Upstate Biotechnology, Lake Placid, New York) as template. The PCR product was then inserted into the pEGFP $\mathrm{N}-1$ vector (Clontech, Palo Alto, California) upstream of sequences encoding the GFP (termed as pRafGFP). The ErbB2 (America Type Culture Collection 


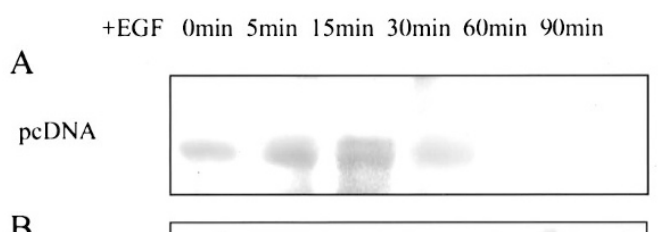

B

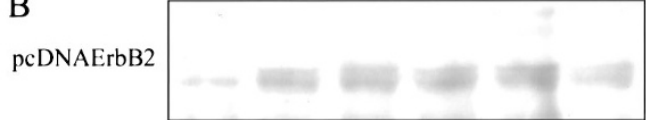

Blot: anti-phospho-Raf-1
$0 \mathrm{~min} 5 \mathrm{~min} 15 \mathrm{~min} 30 \mathrm{~min} 60 \mathrm{~min} 90 \mathrm{~min}$
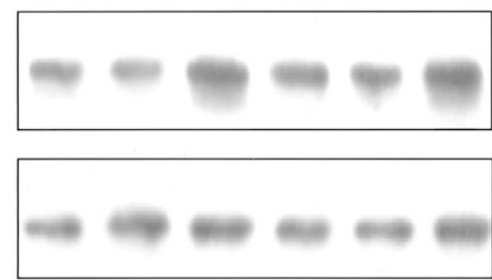

Reblot: anti-actin

C

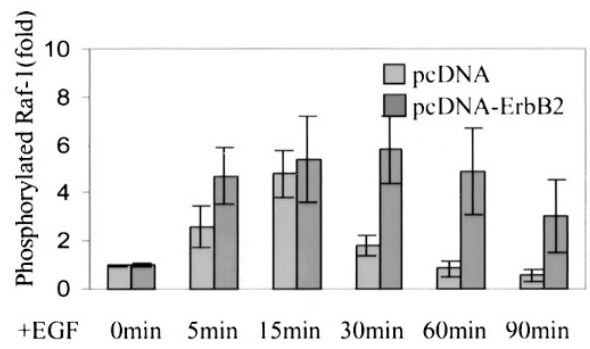

+ EGF $0 \mathrm{~min} 5 \mathrm{~min} 15 \mathrm{~min} 30 \mathrm{~min} 60 \mathrm{~min} 90 \mathrm{~min}$
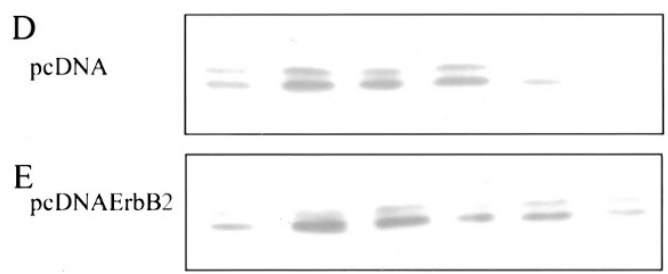

Blot: anti-phospho-MAPK

$0 \mathrm{mim} 5 \mathrm{~min} 15 \mathrm{~min} 30 \mathrm{~min} 60 \mathrm{~min} 90 \mathrm{~min}$
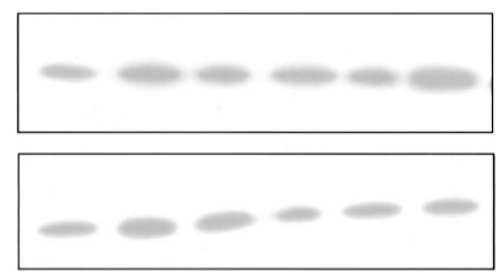

Reblot: anti-MAP kinase-1

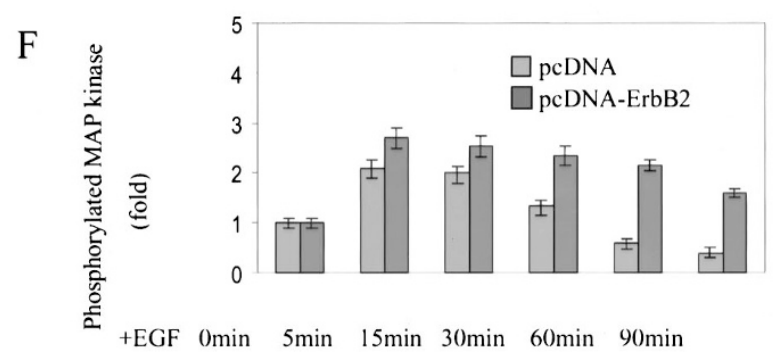

Figure 4.

Immunoblot analysis of activated Raf-1 and activated MAP kinase in BT20 cells transfected by pcDNA or pcDNAErbB2. Total lysates of BT20 cells, transfected with pcDNA or pcDNAErbB2 were subjected to immunoblot analysis with the polyclonal rabbit anti-phospho-Raf-1 (A and B, left) and reblotted with the monoclonal mouse anti-actin antibody ( $A$ and B, right). The relative intensity of phosphorylated Raf-1 was determined by densitometric analysis of autoradiographs (C). Transfected BT-20 cells were also subjected to immunoblot analysis with the anti-phospho-p42/44 MAP kinase ( $D$ and $E$, left) and reblotted with the monoclonal mouse anti-MAP kinase antibody ( $\mathrm{D}$ and $\mathrm{E}$, right). The relative intensity of phosphorylated MAP kinase was determined by densitometric analysis of autoradiographs (F).

[ATCC], Rockville, Maryland) cDNA was inserted into the pcDNA3.1 vector (termed pcDNAErbB2).

\section{Cell Lines}

BT20 and SKBR3 breast cancer cell lines (ATCC) were maintained in Dulbecco's modified essential medium (DMEM; Canadian Life Technologies, Burlington, Ontario) supplemented with 10\% FCS (Hyclone; PDI Biosciences, Aurora, Ontario) and $100 \mu \mathrm{g} / \mathrm{ml}$ streptomycin and $100 \mathrm{U} / \mathrm{ml}$ penicillin (Canadian Life Technologies). Cells were transiently transfected with the indicated plasmid DNA using lipofectamine (Canadian Life Technologies) one day before being used in experiments.

\section{Subcellular Fractionation}

The isolation of plasma membrane (PM) and endosomal (EN) fractions was performed using a protocol modified from Di Guglielmo et al (1994). Two 150 mm-diameter plates were used for each condition. Cells were grown to $80 \%$ confluence and starved by incubation in serum-free medium overnight. The cells were then treated with $100 \mathrm{ng} / \mathrm{ml}$ EGF (Upstate Biotechnology) for the indicated times at $37^{\circ} \mathrm{C}$. Cell monolayers were harvested and homogenized in 2.0 $\mathrm{ml}$ of homogenization buffer $(0.25 \mathrm{M}$ sucrose, $20 \mathrm{~mm}$ Tris- $\mathrm{HCl}, \mathrm{pH} 7.5,1 \mathrm{~mm} \mathrm{MgCl}_{2}, 4 \mathrm{~mm} \mathrm{NaF}$ ) containing protease inhibitors (Roche Diagnostics, Laval, Que- 
bec). The homogenates were centrifuged at $280 \times g$ for 5 minutes to pellet cell debris and nuclei (P1). The first supernatant (S1) was centrifuged at $1,500 \times g$ for 10 minutes to yield a second supernatant (S2) and a pellet (P2). The S2 fraction was centrifuged at 200,000 $\times g$ for 30 minutes to yield a supernatant corresponding to the cytosolic (Cyt) fraction. The pellet was resuspended in $1.15 \mathrm{M}$ sucrose in homogenization buffer, overlaid with $1.0 \mathrm{~m}$ sucrose and $0.25 \mathrm{~m}$ sucrose cushions, and centrifuged at $200,000 \times g$ for 60 minutes. The EN fraction was collected at the 0.25 to $1.00 \mathrm{~m}$ sucrose interface. P2 was resuspended in homogenization buffer and overlaid on a $1.42 \mathrm{~m}$ sucrose cushions and centrifuged at $82,000 \times g$ for 30 minutes. The pellicle at the 0.25 to $1.42 \mathrm{~m}$ interface was collected, the sucrose concentration diluted to $0.39 \mathrm{~m}$, and the suspension centrifuged at $1,500 \times g$ for 15 minutes to obtain a pellet corresponding to the PM fraction.

\section{Immunoblot Analysis}

BT-20 cells and BT-20 cells transiently transfected with pcDNAErbB2 or pcDNA3.1 were harvested by scraping into RIPA lysis buffer. Cell lysates (30 $\mu \mathrm{g}$ protein/lane) were subjected to immunoblot analysis to determine the levels of EGFR, ErbB2, phosphoMAP kinase, and phospho-Raf- 1 . The subcellular distribution of Raf-1 was determined by immunoblot analysis of subcellular fractions isolated from BT-20 cells or transiently transfected BT-20 cells. Subcellular fractions containing $20 \mu \mathrm{g}$ of protein were subjected to electrophoresis on $10 \%$ polyacrylamide gels containing SDS and electrophoretically transferred onto nitrocellulose membranes. The nitrocellulose membranes were blocked by incubation in 3\% BSA in Tris-buffered saline containing $0.5 \%$ Tween-20 (TBST). The different membranes were then probed with polyclonal goat anti-ErbB2 (Santa Cruz Biotechnology, Santa Cruz, California), polyclonal goat antiEGFR (Santa Cruz Biotechnology), monoclonal mouse anti-Raf-1 (Transduction Laboratories, Lexington, Kentucky), polyclonal rabbit anti-phospho-Tyr340/ 341Raf-1 (Biosource Inc., Montreal, Quebec), or polyclonal rabbit anti-phospho-p42/44 MAP kinase (New England Biolabs, Beverly, Massachusetts) in 0.5\% BSA in TBST. The primary antibodies were detected by incubation in polyclonal goat anti-rabbit IgG coupled to HRP, polyclonal donkey anti-goat IgG coupled to HRP, or polyclonal goat anti-mouse IgG coupled to HRP (Santa Cruz Biotechnology). HRP was detected by chemiluminescence using the Supersignal reagent (Pierce Chemical, Rockford, Illinois) and detected with ECL X-Ray film (Amersham Pharmacia Biotech, Oakville, Ontario, Canada).

The nitrocellulose membranes were stripped by incubating in stripping buffer (1\% SDS, $100 \mathrm{~mm}$ $\beta$-mercaptoethanol in TBS) for 30 minutes at room temperature and then washed in TBS at least six times. The nitrocellulose membrane was then used for reblotting with monoclonal mouse anti-Erk-1 (Transduction Laboratories), monoclonal mouse anti-actin
(Santa Cruz Biotechnology), polyclonal goat antiErbB2, or polyclonal goat anti-EGFR.

\section{Immunofluorescence Microscopy}

Cells were grown on glass coverslips to subconfluence and serum-starved overnight. The cells were treated with EGF $(100 \mathrm{ng} / \mathrm{ml})$ for the indicated times, fixed by incubation in cold methanol for 5 minutes, and then washed with PBS. The cells were then permeabilized by incubation in $0.2 \%$ Triton $X-100$ in PBS for 10 minutes and blocked by incubation in 3\% BSA in PBS for 30 minutes. The cells were incubated with polyclonal goat anti-EGFR antibody at room temperature for 1 hour. The coverslips were washed three times with PBS and then incubated with donkey anti-goat IgG conjugated with rhodamine. For detection of GFP-dependent fluorescence, the transiently transfected cells were fixed by incubation in cold methanol for 5 minutes and then washed with PBS. Samples were visualized under an oil immersion lens using a Zeiss Axiophot fluorescence microscope (Carl Zeiss, Gottinger, Germany) and digital images captured using Northern Eclipse computer software.

\section{References}

Afify AM, Werness BA, and Mark HF (1999). HER-2/neu oncogene amplification in stage I and stage III ovarian papillary serous carcinoma. Exp Mol Pathol 66:163-169.

Bitran JD, Samuels B, Trujillo Y, Klein L, Schroeder L, and Martinec $J$ (1996). Her2/neu overexpression is associated with treatment failure in women with high-risk stage II and stage IIIA breast cancer ( $>10$ involved lymph nodes) treated with high-dose chemotherapy and autologous hematopoietic progenitor cell support following standard-dose adjuvant chemotherapy. Clin Cancer Res 2:1509-13.

Cantley LC, Auger KR, Carpenter C, Duckworth B, Graziani A, Kapeller R, and Soltoff S (1991). Oncogenes and signal transduction. Cell 64:281-302.

Carpenter $G$ (1987). Receptors for epidermal growth factor and other polypeptide mitogens. Annu Rev Biochem 56:881-914.

Crews CM and Erikson RL (1993). Extracellular signals and reversible protein phosphorylation: What to Mek of it all. Cell 74:215-217.

Dent P, Reardon DB, Wood SL, Lindorfer MA, Graber SG, Garrison JC, Brautigan DL, and Sturgill TW (1996). Inactivation of raf- 1 by a protein-tyrosine phosphatase stimulated by GTP and reconstituted by Gai/o subunits. J Biol Chem 271:3119-3123.

Di Guglielmo GM, Baass PC, Ou WJ, Posner BI, and Bergeron JJ (1994). Compartmentalization of SHC, GRB2 and mSOS, and hyperphosphorylation of Raf-1 by EGF but not insulin in liver parenchyma. EMBO J 13:4269-4277.

Earp HS, Dawson TL, Li X, and Yu H (1995). Heterodimerization and functional interaction between EGF receptor family members: A new signaling paradigm with implications for breast cancer research. Breast Cancer Res Treat 35:115-132.

Goldman R, Levy RB, Peles E, and Yarden Y (1990). Heterodimerization of the erbB-1 and erbB-2 receptors in human breast carcinoma cells: A mechanism for receptor transregulation. Biochemistry 29:11024-11028. 
Graus-Porta D, Beerli RR, and Hynes NE (1995). Single-chain antibody-mediated intracellular retention of ErbB-2 impairs Neu differentiation factor and epidermal growth factor signaling. Mol Cell Biol 15:1182-1191.

Heidecker G, Huleihel M, Cleveland JL, Kolch W, Beck TW, Lloyd P, Pawson T, and Rapp UR (1990). Mutational activation of C-raf-1 and definition of the minimal transforming sequence. Mol Cell Biol 10:2503-2512.

Heldin CH (1995). Dimerization of cell surface receptors in signal transduction. Cell 80:213-223.

Holmes WE, Sliwkowski MX, Akita RW, Henzel WJ, Lee J, Park JW, Yansura D, Abadi N, Raab H, Lewis GD, and et al (1992). Identification of heregulin, a specific activator of p185erbB2. Science 256:1205-1210.

Ikawa S, Fukui M, Ueyama Y, Tamaoki N, Yamamoto T, and Toyoshima K (1988). B-raf, a new member of the raf family, is activated by DNA rearrangement. Mol Cell Biol 8:2651-2654.

Janes PW, Daly RJ, deFazio A, and Sutherland RL (1994). Activation of the Ras signaling pathway in human breast cancer cells overexpressing erbB-2. Oncogene 9:36013608.

Karunagaran D, Tzahar E, Beerli RR, Chen X, Graus-Porta D, Ratzkin BJ, Seger R, Hynes NE, and Yarden Y (1996). ErbB-2 is a common auxiliary subunit of NDF and EGF receptors: Implications for breast cancer. EMBO J 15:254-264.

King CR, Borrello I, Bellot F, Comoglio P, and Schlessinger J (1988). EGF binding to its receptor triggers a rapid tyrosine phosphorylation of the erbB-2 protein in the mammary tumor cell line SK-BR-3. EMBO J 7:1647-1651.

Lacroix H, Iglehart JD, Skinner MA, and Kraus MH (1989). Overexpression of erbB-2 or EGF receptor proteins present in early stage mammary carcinoma is detected simultaneously in matched primary tumors and regional metastases. Oncogene 4:145-151.

Lange-Carter CA, Pleiman CM, Gardner AM, Blumer KJ, and Johnson GL (1993). A divergence in the MAP kinase regulatory network defined by MEK kinase and Raf. Science 260:315-319.

Leevers SJ, Paterson HF, and Marshall CJ (1994). Requirement for Ras in Raf activation is overcome by targeting Raf to the plasma membrane. Nature 369:411-414.

Lenferink AE, Pinkas-Kramarski R, van de Poll ML, van Vugt MJ, Klapper LN, Tzahar E, Waterman H, Sela M, van Zoelen EJ, and Yarden $Y$ (1998). Differential endocytic routing of homo- and hetero-dimeric ErbB tyrosine kinases confers signaling superiority to receptor heterodimers. EMBO $\mathrm{J} 17$ : 3385-3397.

Lonn U, Lonn S, Friberg S, Nilsson B, Silfversward C, and Stenkvist B (1995). Prognostic value of amplification of c-erb-B2 in bladder carcinoma. Clin Cancer Res 1:1189-1194.

Marshall CJ (1994). Signal transduction. Hot lips and phosphorylation of protein kinases. Nature 367:686.

Mineo C, James GL, Smart EJ, and Anderson RG (1996). Localization of epidermal growth factor-stimulated Ras/Raf-1 interaction to caveolae membrane. J Biol Chem 271:1193011935.

Moelling K, Heimann B, Beimling P, Rapp UR, and Sander T (1984). Serine- and threonine-specific protein kinase activities of purified gag-mil and gag-raf proteins. Nature 312:558-561.
Morrison DK and Cutler RE (1997). The complexity of Raf-1 regulation. Curr Opin Cell Biol 9:174-179.

Nakajima M, Sawada H, Yamada Y, Watanabe A, Tatsumi M, Yamashita J, Matsuda M, Sakaguchi T, Hirao T, and Nakano $H$ (1999). The prognostic significance of amplification and overexpression of c-met and c-erbB-2 in human gastric carcinomas. Cancer 85:1894-1902.

Polkowski W, van Sandick JW, Offerhaus GJ, ten Kate FJ, Mulder J, Obertop H, and van Lanschot JJ (1999). Prognostic value of Lauren classification and c-erbB-2 oncogene overexpression in adenocarcinoma of the esophagus and gastroesophageal junction. Ann Surg Oncol 6:290-297.

Ross JS and Fletcher JA (1999). HER-2/neu (c-erb-B2) gene and protein in breast cancer. Am J Clin Pathol 112:S53-67.

Ross JS, Yang F, Kallakury BV, Sheehan CE, Ambros RA, and Muraca PJ (1999). HER-2/neu oncogene amplification by fluorescence in situ hybridization in epithelial tumors of the ovary. Am J Clin Pathol 111:311-316.

Satoh T, Nakafuku M, and Kaziro Y (1992). Function of Ras as a molecular switch in signal transduction. J Biol Chem 267:24149-24152.

Schwartz S Jr, Caceres C, Morote J, De Torres I, RodriguezVallejo JM, Gonzalez J, and Reventos J (1999). Gains of the relative genomic content of erbB-1 and erbB-2 in prostate carcinoma and their association with metastasis. Int $\mathrm{J}$ Oncol 14:367-371.

Shimizu K, Nakatsu Y, Sekiguchi M, Hokamura K, Tanaka K, Terada M, and Sugimura T (1985). Molecular cloning of an activated human oncogene, homologous to v-raf, from primary stomach cancer. Proc Natl Acad Sci USA 82:5641-5645.

Slamon DJ, Clark GM, Wong SG, Levin WJ, Ullrich A, and McGuire WL (1987). Human breast cancer: Correlation of relapse and survival with amplification of the HER-2/neu oncogene. Science 235:177-182.

Slamon DJ, Godolphin W, Jones LA, Holt JA, Wong SG, Keith DE, Levin WJ, Stuart SG, Udove J, Ullrich A (1989). Studies of the HER-2/neu proto-oncogene in human breast and ovarian cancer. Science 244:707-712.

Sorkin A and Waters CM (1993). Endocytosis of growth factor receptors. Bioessays 15:375-382.

Stanton VP Jr, Nichols DW, Laudano AP, and Cooper GM (1989). Definition of the human raf amino-terminal regulatory region by deletion mutagenesis. Mol Cell Biol 9:639-647.

Tan M, Yao J, and Yu D (1997). Overexpression of the c-erbB-2 gene enhanced intrinsic metastasis potential inhuman breast cancer cells without increasing their transformation abilities. Cancer Res 57:1199-1205.

Vojtek AB, Hollenberg SM, and Cooper JA (1993). Mammalian Ras interacts directly with the serine/threonine kinase Raf. Cell 74:205-214.

Wada T, Qian XL, and Greene MI (1990). Intermolecular association of the p185 neu protein and EGF receptor modulates EGF receptor function. Cell 61:1339-1347.

Wang Z, Zhang L, Yeung TK, and Chen X (1999). Endocytosis deficiency of epidermal growth factor (EGF) receptor-ErbB2 heterodimers in response to EGF stimulation. Mol Biol Cell 10:1621-1636. 